\title{
LOCALIZATION OF PAIN ACCOMPANYING FARADIC EXCITATION OF STOMACH AND DUODENUM IN HEALTHY INDIVIDUALS ${ }^{1}$
}

\author{
By EDWARD A. BOYDEN AND LEO G. RIGLER
}

(From the Departments of Anatomy and Radiology, University of Minnesota, Minneapolis)

(Received for publication June 15, 1934)

The observations recorded in this article were originally by-products of a group of experiments designed to test whether or not the human gallbladder is subject to inhibitory reflexes originating in the gastro-intestinal tract (1). Subsequently, these experiments were repeated and elaborated in the belief that pain originating from ring contraction of the gut might be more specifically localized than sensations arising from inflamed or distended surfaces of the hollow viscera, and so throw additional light on the baffling problem of splanchnic pain.

\section{METHODS}

The method of investigation consisted of sending an induction current through a Rehfuss tube, the metal end of which had been converted into an electrode and swallowed to the desired depth. The second electrode was made of a moist felt pad sewed to a copper screen and applied to the arm or leg. The subjects chosen for experimentation were eleven volunteer medical students in the University of Minnesota, who could be depended upon for intelligent and trustworthy cooperation.

The strength of current employed, as measured by the position of the secondary coil over the core of a Harvard inductorium, was similar to that used in ordinary physiological experiments. When the induction coil was attached to two dry cells, the minimal stimulus required to produce visceral sensation varied with the individual but ranged from Position $61 / 2$ to Position 5 , i.e., with the secondary coil from 1 to $2 \frac{1}{2}$ centimeters over the end of the core. The maximum stimulus used (Position 4) was of a strength which was unbearable when applied to the lips, but still tolerated by the gut.

\section{PRELIMINARY OBSERVATIONS}

The effect of the current upon the stomach, as observed under the fluoroscope, was to induce a sphincteric contraction of the gut and then an

\footnotetext{
${ }^{1}$ Aided by a grant from funds of the Graduate School.
} 
increased peristalsis distal to the point of stimulation. Occasionally a whole segment of the gut would contract uniformly (see Figure 4 of article cited (1)). If the electrode was not in contact with the wall of the stomach but merely lay in its cavity, the effect of the current was much reduced. Once, in such a case, peristalsis already in progress was checked by the current. Occasionally, after prolonged experimentation, the subject failed to respond to the stimulus. This was attributed to mucus (afterwards regurgitated with the tube) which apparently collected in such quantities as to insulate the gut against the current; for when the position of the tube or patient was changed, the response to the current was restored.

The effect of the current upon the duodenum could not be ascertained because the barium passed through this portion of the gut so rapidly. It was presumed from animal experimentation, however, that the current caused ring contraction of the intestine also.

In both organs contraction of the visceral musculature was usually accompanied by some degree of abdominal rigidity, depending on the strength of the current. Sometimes this rigidity occurred when the current was too mild to induce any visceral sensation. Also it seemed to be more pronounced when the second electrode was fastened to the arm than when it was applied to the leg.

The nature of the sensation that accompanied contraction of the gut ranged from barely perceptible feelings of pressure, gnawing sensations and heart-burn, to dull and severe colicky pain. Frequently there was a "throbbing" sensation which apparently synchronized with the alternation of the current. When a mild current was employed, one or more seconds usually elapsed before visceral sensations were felt. Then the pain increased gradually to a climax. In the case of very strong currents, causing spastic contraction of the gut, the pain was immediate.

Localization of these sensations was characterized by two general features: (1) the depth of the sensation (it seeming to come from well beneath the abdominal wall); (2) the definiteness with which it could be located in the upper quadrants of the abdomen (the subject always pointing to the spot with one finger).

\section{The localization of pain}

1. Posture constant. The first group of experiments (Figure 1) represent a summary of observations made upon one student on six different days scattered over a period of several months. Figure 2 records incidental observations made upon five other students in connection with gallbladder experiments. In each case the subject was lying prone on the $x$-ray table-the position in which stomach and duodenum approach nearest to the $\mathrm{x}$-ray plate. The circles in each figure indicate the position of the electrode in the gut as determined by $\mathbf{x}$-ray films taken immediately 
before the current was applied. The dots indicate the area on the abdominal wall to which the subject pointed immediately after the current was interrupted.

A cursory examination of these figures shows two apparently contradictory features: a tendency for the pain areas to follow the course of the

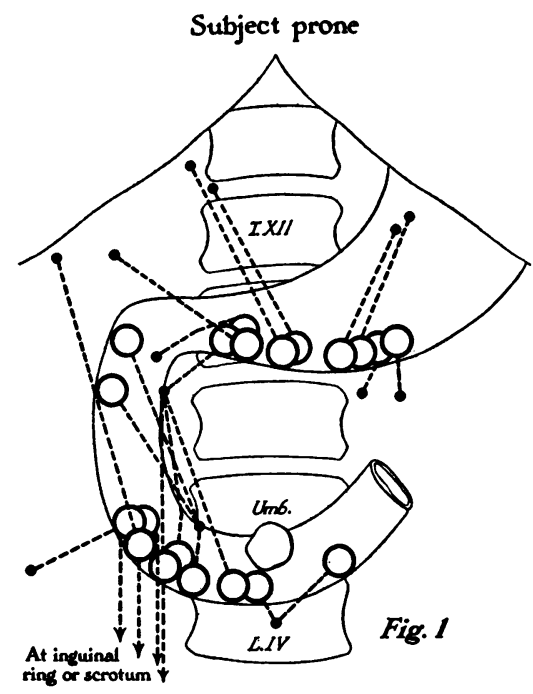

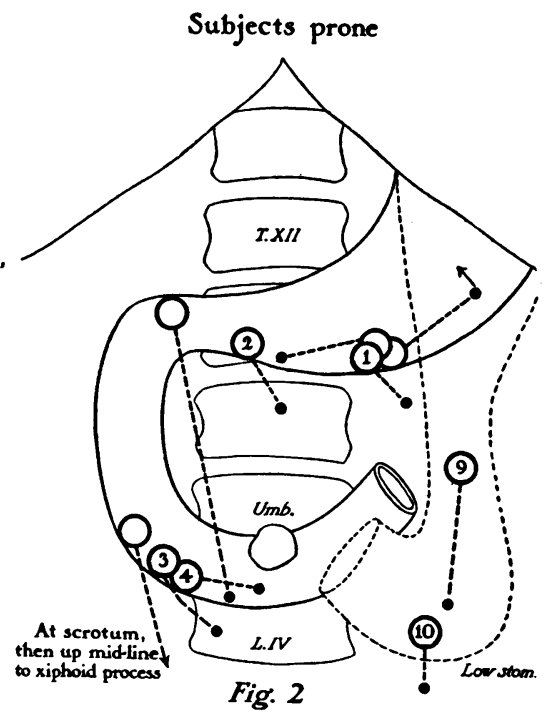

Fig. 2

Fig. 1. Assembly Drawing Showing Localization of Pain in Same Subject on Six Different Days (Series II-VII, Case E. F. M.)

In each experiment faradic stimulation of stomach or duodenum was applied for a period of 10 seconds. Circles indicate position of electrode in gut as determined by $\mathrm{x}$-ray films; dots, the position on abdominal wall pointed to by subject as site of pain. For other details, see Figures 3 to 6 .

\section{Fig. 2. Localization of Pain in Five Other Normal Subjects}

Same technique employed as before. 1 to 4 , four successive readings made from one subject, Case A. M. L. (March 8, 1933) : 1, 9:50 a.m., weak current, initial pressure sensation suddenly changing to "feeling as if a bubble had burst" or as if subject had been "hit with a blow"; $2,10: 16$, strong current, sharp colic increasing in intensity, "pretty bad"; $3,10: 47$, strong current, "extreme colic"; 4, $11: 02$, moderate current, dull ache increasing to sharp colic. 9 and 10, two readings from Case D. S. F. (February 27, 1933): 9, 9:51 a.m., weak current, feeling of pressure; 10,10:20, strong current, dull pain.

stomach and duodenum-so that if certain dots were selected they would outline approximately the position of the intestinal tube; and a certain aberrancy whereby pain originating in the stomach is sometimes referred to the left or to the right border of the ribs instead of to the overlying region, while pain from the right upper duodenal flexure may be projected downward to the right side of the umbilicus; or pain from the right lower 
duodenal flexure may appear in the right epigastrium or at the inguinal region. ${ }^{2}$

A good illustration of the apparent tendency of gastroduodenal pain to follow the course of the electrode is shown in Experiments 1 to 4 (Figure 2)-four readings from the same individual (A. M. L.) taken at 15 to 30 minute intervals. Even more striking is the case of a student with a low-lying stomach (Figure 2) that projected an inch or more below the umbilicus when the subject was prone and the stomach empty. ${ }^{3}$ In this subject (D. S. F.) dull pain induced by stimulation of the upper limb of the stomach was not localized in the left epigastrium, but in the left umbilical zone (Experiment 9, Figure 2); and when, fifteen minutes later, the electrode was swallowed another two inches, to the bottom of the greater curvature, the pain area descended with it (Experiment 10)..$^{4}$

Observations such as these, seemed at first to render it unlikely that we were dealing with pain that was being referred from the viscera to the abdominal wall, because no matter what the position of this ptotic stomach was, its nerve supply should be the same as that of any other stomach, and so the pain should have been referred to zones of the 6th to 9th thoracic nerves. Instead it was localized in the territory supplied by the 11th thoracic nerve. This case seemed to indicate, therefore, that we were dealing either with true visceral pain-i.e., with pain directly felt in the wall of the stomach-or else with excitation of the anterior parietal peritoneum-as recently predicated by Morley (3).

This author reported, for instance, that the site of deep tenderness in acute obstructive cholecystitis descended with increasing distention (and consequent elongation) of the biliary vesicle; and that the area of deep tenderness in ulcer patients followed the change in position of the stomach or duodenum. He interpreted such gastric pains as being due either to mechanical or chemical stimulation of the anterior parietal peritoneum, which was not felt in the peritoneum but was localized in the immediately overlying skin-a so-called peritoneo-cutaneous radiation.

2. Effect of change in posture. Impressed with Morley's account we undertook to see how change of position would affect the site of pain. Employing the same subject as before, it was soon noted that the pain area

2 In the latter case, the sensations were mostly "quiverings" in the territory of the cremasteric muscle and so may have been due to a spreading of the current to the ureter or internal spermatic vessels, which lie just deep to the thin posterior wall of the duodenum. This seems the more probable since the spermatic cord is not affected by experimental distention of this part of the duodenum (Fig. 11).

3 This is an extreme type, apparently falling within the small group which Moody (2) describes as occurring in 3.2 per cent of normal male students (cf. Figure 7 of article cited).

4 Roentgenograms showing the exact position of the electrodes in this case may be seen in Figure 12, Boyden and Rigler (1). 

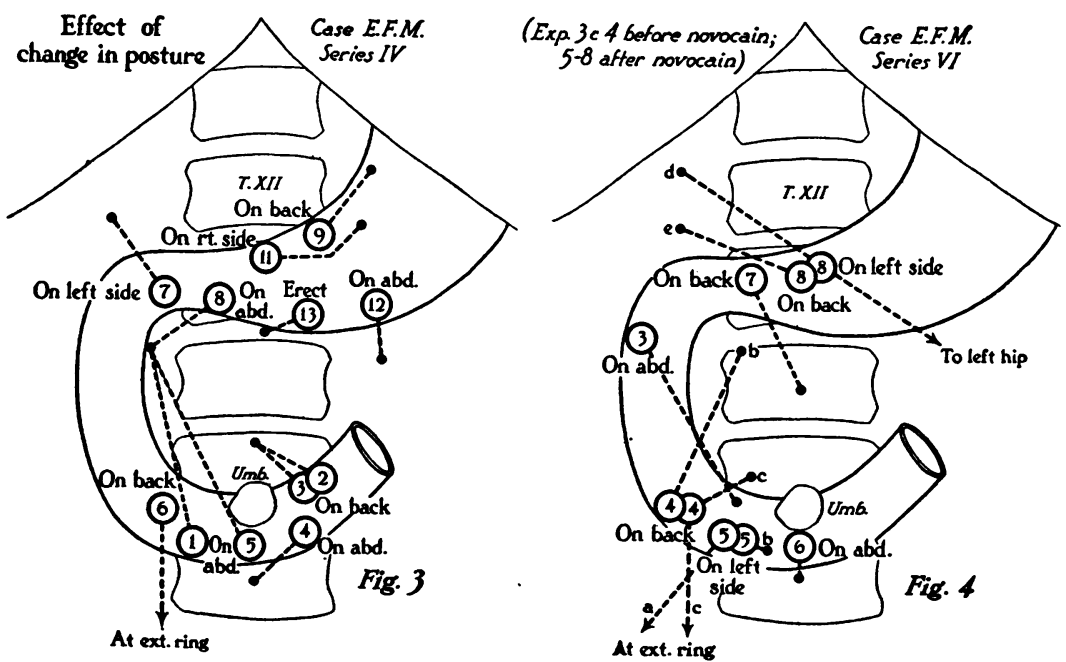

Fig. 3. Group of Experiments Illustrating Effect of Change of Posture on Site of Pain. Case E. F. M., Series IV (January 21, 1934)

Circles indicate position of electrode; dots indicate site of pain; arabic numerals within circles, the sequence of experiments : 1, 12:02 p.m., weak current, "dull muscular pull"; $2,12: 08$, weak current, dull ache, stronger sensation than at $1 ; 3,12: 11$, strong current, pain more severe, but still dull; seemed to "flutter" with buzzer of induction apparatus and to "pull toward the back"; 4 , $12: 24$, strong current, sharp colic, vibrating but stationary; $5,12: 38$, moderate current, dull pain; $6,12: 44$, moderate current, dull ache in region of spermatic cord, "felt like cremasteric muscle"; 7, $1: 03$, moderate current, sharp pain: " not crampy but like line of pain"; $8,1: 08$, moderate current, dull cramp; 9, $1: 16$, moderate current, dull "spasm"; $11,1: 25$, moderate current, dull spasm; $12,1: 30$, moderate current, colicky pain; $13,1: 35$, moderate current, faint flutter.

\section{Fig. 4. Group of Experiments Extending Observations Shown in Figure}

3. (Originally Designed to Test Effect of Thoracic Nerve Block.) Case E. F. M., Series VI (Ferruary 15, 1934)

Arabic numerals indicate sequence of experiments : 3, 7:50 a.m., strong current, dull sensation not painful ; $4 b, 7: 55$, weak current for 5 seconds only, painless "tugging" inside; 4c, 7:57, weak current for usual 10 seconds, sharp pain starting slowly and increasing to steady colicky pain (felt after current stopped), followed by "fluttering in spermatic cord." $8: 23$ to $9: 45$ a.m., injections of $400 \mathrm{cc}$. of 1 per cent novocain deep in intercostal spaces 6 to 11 , also along infracostal borders, also subcutaneously, in unsuccessful attempt to anesthetize whole anterior abdominal wall; subject subsequently found to be four times as resistant to novocain as average individual; $5 a, 9: 48$, strong current, deep pain getting worse; $5 b, 9: 50$, strong current, sharp pain "worse" than $5 a$; $6,9: 56$, strong current, severe colicky pain (all that subject could stand); 7 , $10: 02$, moderate current, dull pain; $8 a$ (on abdomen), $b$ (on right), $c$ (on left), no feeling with strong current; $8 d$, strong current, $10: 12$, dull sensation (plus twitching over left tensor fasciae latae); $8 e, 10: 14$, strong current, barely noticeable sensation. (The lessened pain noted in Experiments 7 to 10 may have been due to the accumulation of mucus in the stomach, or to general narcosis caused by large amount of novocain administered.) 
frequently (but not always) shifted when the subject turned onto his side or back or stood erect. Thus starting with the patient supine (Experiment 9 , Figure 3 ) a shift of the body onto the right side lowered the pain area (Experiment 11); a rolling over onto the abdomen still further lowered it (Experiment 12); and a standing posture swung the pain area to the midline, but not as far down as one would expect (Experiment 13). Similarly, shifting the subject from back to abdomen (with consequent lowering of the duodenum) shifted the area of localization from above to below the umbilicus (Experiments 2 to 4, Figure 3; Experiments 4 and 5, Figure 4). These cases illustrate the tendency of the pain to follow the change in position of the gut. In Figure 5, however, the opposite tendency is recorded. Here (Experiments 2 and 3 ) a change from prone to supine position shifted the pain from high up in the epigastrium to the umbilicus, just the reverse of the movement of the gut. Thus not all observations were consistent with Morley's theory. This caused us to test it by other methods.

3. Experiments designed to test the rôle of the parietal peritoneum. Believing, on a priori grounds, that the current was not strong enough to penetrate the hollow viscus and still stimulate nerve endings in the anterior parietal peritoneum (a distance of several inches from the electrode) deep manual pressure was exerted over the lower end of the duodenum. This should have increased the pain by bringing the parietal peritoneum nearer to the area of current density. Yet no such increase of pain was noted.

Again, peritoneal pain should have been in the nature of a sharp stitch and should always have appeared immediately over the electrode-witness the experiments dealing with direct mechanical irritation of the anterior peritoneum (Capps and Coleman (4)). Yet in twelve cases in which the electrode was in that part of the stomach that lies against the anterior wall -and with the patient prone-the sensations reported were not stitch-like pains but pressure or burning sensations, dull or colicky pain; nor were they localized accurately enough to meet the above requirements for stimulation of the parietal peritoneum.

Furthermore, if the current were spreading from the gut, its direction should have changed when the second electrode was moved-say from the left forearm to the right calf (e.g., Experiments 2c to 2e, Figure 6)-yet moving the second electrode did not change the site of the pain. Accordingly, it was concluded from these experiments that the current could not have directly stimulated the anterior peritoneum.

4. Apparent conditioning of nervous pathways. While the above experiments were being conducted, a subject was encountered in which no modification of external or internal conditions seemed to change the site of pain. Thus when the subject was shifted from a prone to supine position and back again (Experiments 2 to 6, Figure 7)-the electrode remaining 

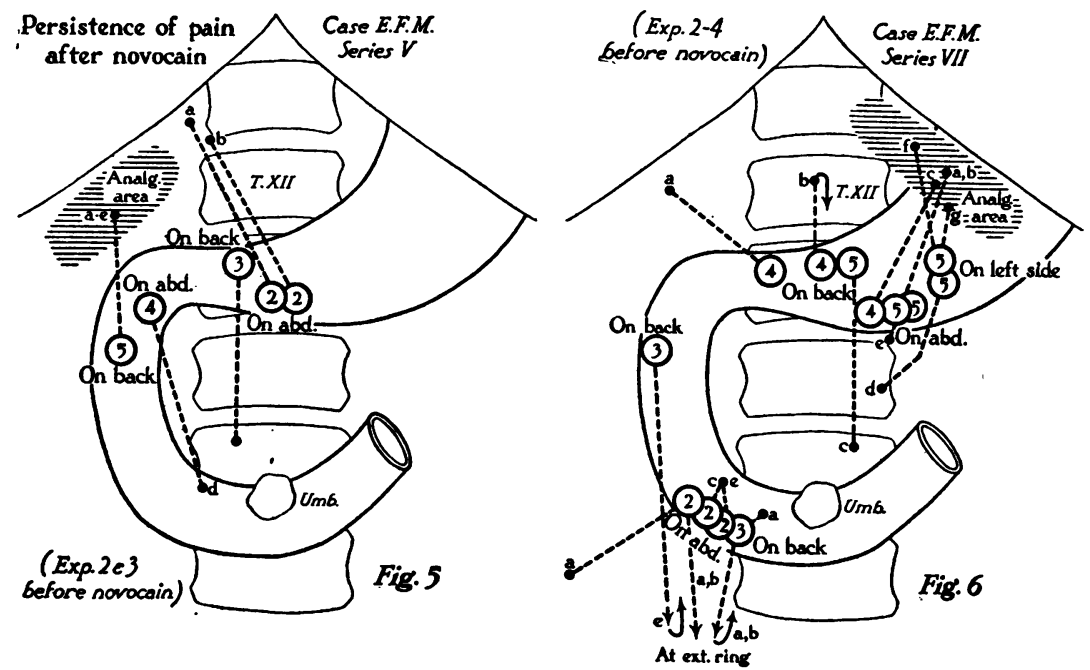

Fig. 5. Group of Experiments Illustrating Persistence of Visceral

Pain under Area of Skin that Had Been Rendered Analgesic to Pin Pricks. Case E. F. M., Series V (February 10, 1934)

Arabic numerals indicate sequence of experiments: $2 a, 7: 47$, weak current, gnawing sensation; $2 b, 7: 49$, strong current, gnawing sensation; $3,7: 54$, strong current, sharp, crampy pain, sort of "gone" feeling. 7:55 to 8:20, subcutaneous, wheal infiltration of $1 / 2$ per cent novocain (and adrenalin) along right and left subcostal border; $4 a-c$, not felt; $4 d, 8: 32$, moderate current, barely felt; $5 a$ and $b, 8: 35-: 37$, moderate current, dull tug, "pulling toward diaphragm"; $5 c$, 8:39, weak current, dull sensation; $5 d$ and $e, 8: 41-: 43$, moderate current, dull sensation stronger than at $5 c$, but not painful. (Note that in Experiments $5 a-e$, a sensation was felt under an area analgesic to pin pricks.)

Fig. 6. Group of Experiments Illustrating Effect of Changing Position of Second Electrode and of Deep Manual Pressure Over First

Electrode. Case E. F. M., Series VII (March 3, 1934)

Arabic numerals indicate sequence of experiments : $2 a, 7: 36$ a.m., moderate current (2d electrode on left calf), dull vibrating pain barely felt, also flutter over right external ring; $2 b, 7: 40$, strong current, "cremasteric flutter"; $2 c$, $7: 43$, moderate current (2d electrode on left forearm), dull pain; $2 e, 7: 52$, moderate current (2d electrode on right calf), dull vibrating sensation; $3 a, 8: 01$, moderate current (2d electrode on right calf), dull pain, more marked than in $2 e$, also felt at external ring going deeper as it moves cephalad two inches; slight abdominal rigidity on right side only; $3 b, 8: 03$, moderate current, sensation in spermatic cord region moving up as before; deep pressure on abdominal wall over duodenal electrode caused no change in intensity of spermatic pain nor was any pain noted at umbilical region as before; $3 e, 8: 10$, moderate current (2d electrode on left forearm), dull pain starting at external ring and going deeper as it moved cephalad two inches (no other abdominal pain) ; 4a, 8:18, moderate current, dull pulsating sensation; $4 b, 8: 20$, moderate current, deep, dull vibrating sensation which traveled downward; $4 c, 8: 23$, strong current, dull vibrating sensation. 8:30 to $9: 10$, subcutaneous infiltration of novocain along left subcostal border; $5 a$ and $b, 9: 15-: 17$, strong current, deep dull sensation same as in $4 c$, appearing under area of skin analgesic to pin pricks; $5 c, 9: 19$, very strong current, dull sensation; $5 d, 9: 21$, very strong current, dull sensation; $5 e, 9: 23$, strong current, dull sensation; $5 f$ and $g, 9: 25-: 27$, strong current, same dull sensation. 
all the time in the pyloric antrum of the stomach-the area of colicky pain continued to hover around the umbilicus. Similarly, when the electrode was drawn up from the pyloric antrum into the cardiac region of the stomach (Experiments 6 to 8, Figure 7), pain was still referred to the umbilicus. This was the more surprising since we were dealing with that case of ptotic stomach, in which, previously, the site of pain had descended with the electrode (Figure 2, Experiments 9 and 10). However, after the subject had arisen and walked from the $\mathrm{x}$-ray table to the fluoroscopic room, and some twenty minutes had elapsed, apparently a new judgment was established, for a new site of pain was localized-namely the one approximately over the electrode (Experiment 9, Figure 8).

These observations seemed to point to a conditioning of nervous pathways-to a temporary selection of one out of many avenues; and, in so doing, it destroyed the simple expectancy that pain arising from a given portion of the gut could be projected onto the abdominal wall with any degree of accuracy.

By the process of elimination these conclusions also focussed attention on the possibility that pain in the gut was being projected to the skin of the abdominal wall from the viscera, notwithstanding its apparent deep location. In this, we were directed by the very significant experiments of Weiss and Davis (5). These authors found that in patients suffering from deep yet definitely localized spontaneous pain (accompanying such disorders as gastric ulcer, acute appendicitis, chronic cholecystitis, etc.), intradermal injection of 2 per cent novocain abolished the pain for several hours. Accordingly, we undertook to block out the areas of skin overlying the site of the pain that accompanied electrical excitation of the gut.

5. Effect of anesthetizing the skin. The first attempts are indicated in Figures 5 and 6. On two different days (Experiment 5) pain was observed to persist under areas of the skin that had been infiltrated with novocain. However, as it was subsequently ascertained by pharmacological tests that this particular subject was four times as resistant to novocain as the average medical student, these experiments were not deemed conclusive. Accordingly, they were repeated in another student, the one previously discussed in connection with Figure 7.

This time, with the electrode just above the angular incisure of the stomach, a colicky pain was localized in the left epigastrium (dot, Experiment 9, Figure 8). Then Area I (Figure 8) was injected both intradermally and subcutaneously with 1 per cent novocain (and adrenalin)..$^{5}$ When stimulation was resumed, with the electrode in the same place (Experiment 10), the same degree of pain was felt as before, but this time it

5 The authors are greatly indebted to Dr. Owen Wangensteen, Chief of the Surgical Service in the University Hospital, for his skilful administration of novocain. 


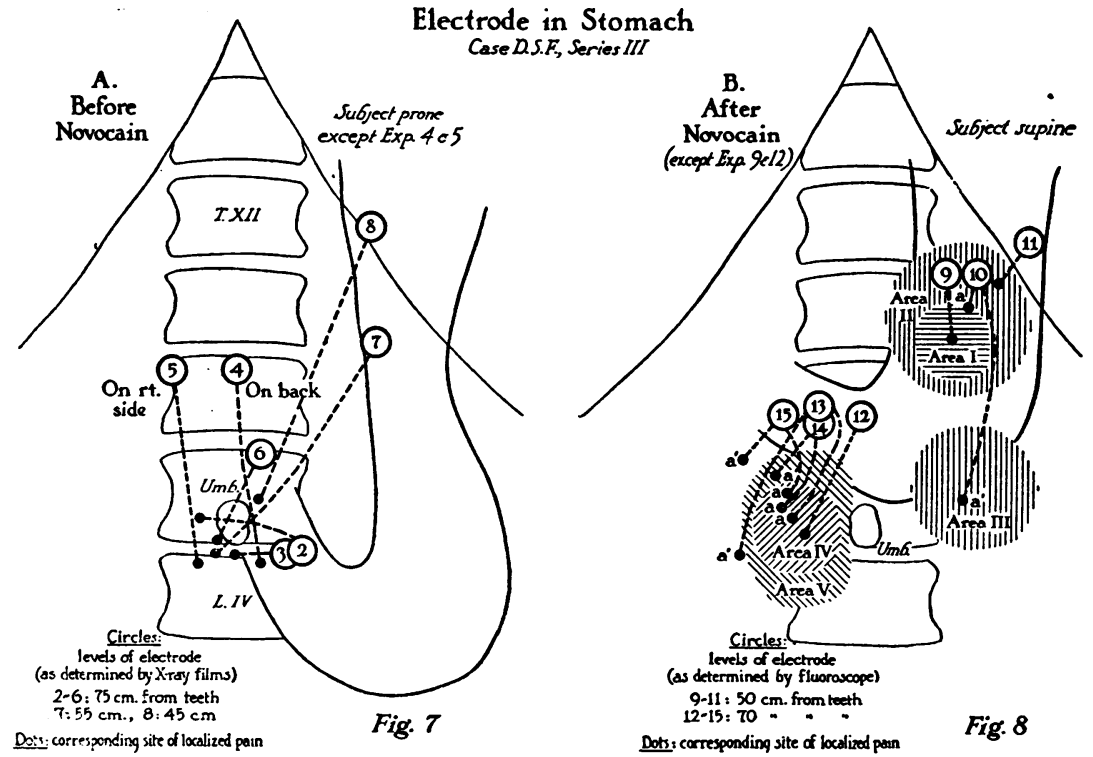

Fig. 7. Group of Experiments Illustrating Failure of Pain Area to Move with Change in Position of Electrode. Case D. S. F., SERIES III (APRIL 16, 1934)

Arabic numerals indicate sequence of experiments: 1 (same position as 2), $7: 16$ a.m., weak current ( $2 \mathrm{~d}$ electrode on left calf), no visceral sensation, yet abdominal rigidity; 2, $7: 21$, moderate current (as before) barely felt; $3,7: 23$, moderate current ( $2 \mathrm{~d}$ electrode on right forearm) ; fluttering sensation stronger than before; $4,7: 30$, moderate current (2d electrode on right forearm), colicky pain ; $5,7: 33$, moderate current (2d electrode on right calf), "knocking" sensation increasing to colic; $6,7: 49$, moderate current (2d electrode on right calf), vibrating sensation painful at end; 7, 7:53, moderate current (2d electrode on left calf), same sensation as at $6 ; 8,8: 05$, moderate current (2d electrode on right calf), unpleasant sensation suggesting nausea but not painful; 9 (Figure $8), 8: 25$, moderate current, colicky pain.

Fig. 8. Series III continued (April 16, 1934): Illustrating the Migration of Pain from Analgesic Areas of the Skin

9, $8: 25$ a.m., moderate current, colicky pain ; 8:30, Area I rendered analgesic by intradermal and subcutaneous injection of 1 per cent novocain; 10, 8:35, moderate current, same sensation as at 9 , but site of pain moved during period of stimulation (10 seconds) from $10 a$ to $a^{\prime} ; 8: 37$, Areas II and III anesthetized; 11 , moderate current, colicky pain felt under Area II ; 12, 8:52, moderate current, colicky pain and throbbing sensation, increasing in intensity; 8:53, Area IV rendered analgesic; $13,8: 58$, moderate current, sensation stronger than before and quite painful, felt simultaneously at $a$ and $a^{\prime} ; 14,8: 59$, ditto; 9:00, Area $V$. anesthetized; 15, $9: 10$, moderate current, same sensation as before : this time felt first at $15 a$, then moved to $a^{\prime}$, though still remaining at $15 a$. 
was localized at the edge of Area I (dot 10a), then traveled during the 10 seconds of the current to position $10 \mathrm{a}^{\prime}$, outside the anesthetized area. This revealed the somewhat startling fact that cutaneous anesthesia had modified the localization of pain and that the skin was involved in sensations arising from spastic contraction of the gut.

Next, Areas II and III were blocked with novocain. This time, with the electrode in the same part of the stomach (Experiment 11) the pain did not migrate but persisted under Area II, thus revealing the existence of a second factor in the recording of visceral pain. Virtually the same results were obtained when the electrode was swallowed as far as the pyloric antrum (Experiment 12). Here, for instance, the pain was localized to the right of the umbilicus. Area IV was then rendered analgesic. Thereupon, pain was localized under Area IV and, simultaneously, outside of it (Experiments 13a and $a^{\prime} ; 14 a$ and $a^{\prime}$ ). Area $V$ was then anesthetized with similar results (Experiment $15 a$ and $a^{\prime}$ ).

\section{DISCUSSION}

The experiments recorded in the preceding pages present seven principal observations regarding the localization of pain arising from electrical excitation of the stomach and duodenum:

1. The sensation is felt deep to the abdominal wall, yet is projected to the skin; 2, the site of pain is restricted to the anterior wall and does not involve the sides or back of the trunk; 3 , within this restricted area it is variable; 4 , it tends to shift with the position of the viscus; 5 , it may stay in one place even when the position of the gut or the point of stimulation is changed; 6 , it migrates from an area of the skin that is anesthetized; 7 , it also persists under an analgesic area.

Superficially viewed, these observations seem to be mutually contradictory. Certainly they demonstrate that the problem of pain which arises from spastic contraction of the gut is not a simple one. Yet it is believed that the interpretation of most of these facts lies within the present bounds of neurologic science.

First, the primary mechanism may be presented with the aid of Figure 9. Let $a$ represent visceral afferent neurones arising in the musculature of the gut and passing via splanchnic nerves and spinal ganglia to thoracic segments VII to IX of the spinal cord, whence the impulse would be relayed to the higher centers and registered in consciousness as true visceral pain-the visceral "muscle sense" of Ryle (6). Simultaneously, impulses from groups of fibers represented by neurone $b$ would pass to the spinal ganglia and there bombard the cell bodies of peripheral neurones $(c)$ which in turn would relay impulses to the higher centers; but 


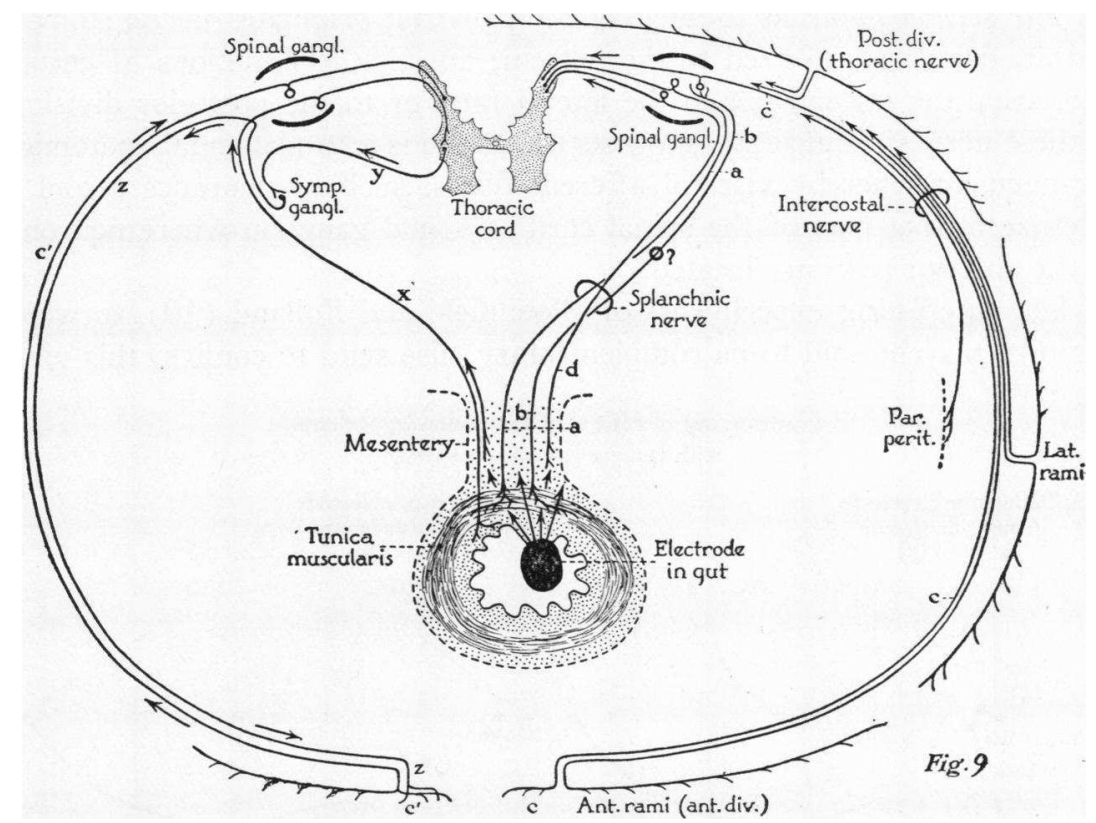

Fig. 9. Diagram Illustrating Probable Course of Pain Impulses

Neurone $a$ : from smooth muscle of gut, via splanchnic nerves and posterior root ganglion to higher centers (protopathic? sense); neurone $b$ : from smooth muscle of gut to synapse around unipolar cells of posterior root ganglion (a viscerocutaneous radiation)-pain appears to come from parietal neurone $c$ (epicritic? sense) and can be modified but not abolished by cutaneous anesthesia; neurone $d$ : from mesenteries; neurones $x, y, z$ (a viscerocutaneous or vasomotor reflex); $x$ : from mucosa or deeper layers of gut to posterior root ganglion and cord; $y$ : preganglionic fiber; $z$ : postganglionic fiber terminating in sensory corpuscles or in blood vessels around nerve endings of parietal neurone $c^{\prime}$, setting up impulses in $c^{\prime}$ that are recorded as hyperalgesia or spontaneous pain from diseased viscera; can be abolished by cutaneous anesthesia.

these impulses would appear to come from the skin. ${ }^{6}$ The resulting localization of pain might be considered to be either a selection of one or the other of these pathways or an integration of impulses from both these sources. This explains why pain may be felt deep to the wall and at the same time be projected to the skin.

- This, of course, does not exclude the older theory that neurones $a$ activate neurones $c$ through synapses in the dorsal horns of the cord. The newer theory, as recently revived by Lemaire (7), is based on Dogiel's discovery of a network of fine branching and anastomosing fibers that surrounds every spinal ganglion cell (8). He believes that such arborizations are the ends or collateral branches of fibers that enter the ganglion through the rami communicantes from the sympathetic nervous system (cf. Ranson (9), Figure 40, c). The location of the cell bodies of such neurones is not known. 
The second point, as to why colicky pain that originates in the stomach and duodenum is referred to the extreme anterior terminations of certain intercostal nerves and not to the lateral rami or to the posterior divisions of these nerves (Figure 9), suggests that there is a fundamental anatomical arrangement whereby visceral afferent fibers, such as neurones $a$ and $b$, arborize in that part of the spinal cord or spinal ganglion where neurones of the anterior rami are located.

The interesting experiments of Bloomfield and Polland (10), to which our own may be said to be complementary, also tend to confirm this view.

\section{Comparison of Effects of Distension (stippled areas) with Faradic Excitation (dots)}
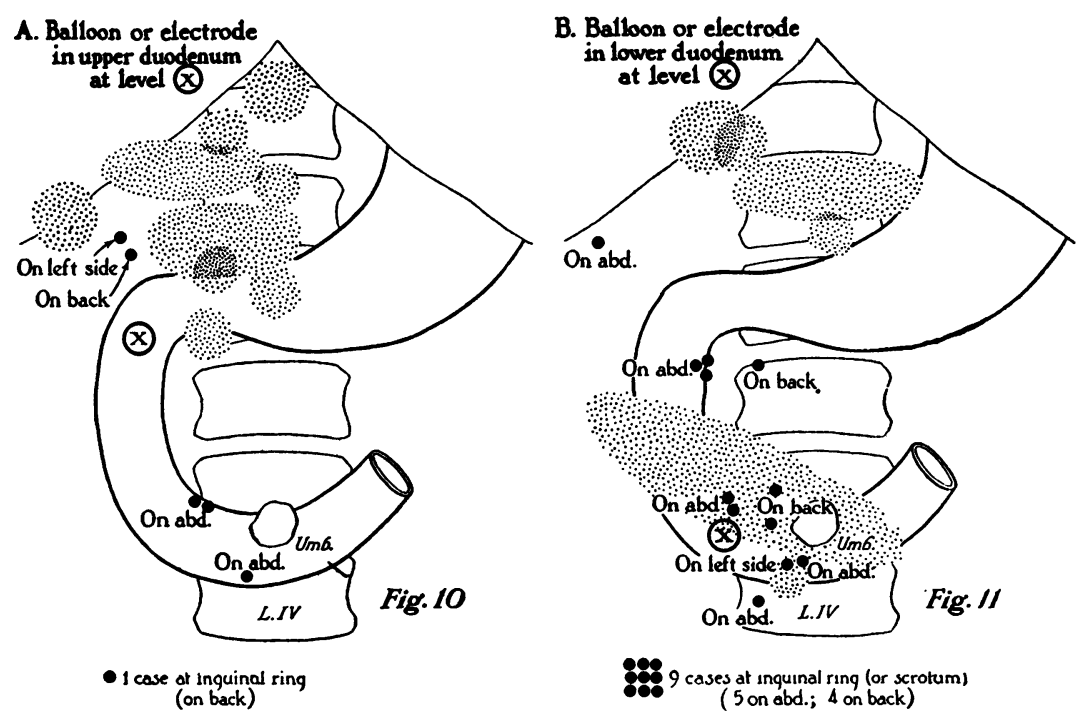

Fig. 10. Diagrams Illustrating Effect of Tension Exerted upon Intestinal Muscle in the Region of the Right Upper Flexure OF THE DUODENum $(x)$

Stippled areas, site of pain following distention of duodenum by the balloon method, with patient erect (Bloomfield and Polland, 1931); dots, site of pain following faradic excitation of same portion of duodenum.

Fig. 11. Diagrams Illustrating Effect of Tension Exerted upon Intestinal Muscle in the Region of the Right Lower Flexure of The Duodenum $(x)$

Stippled areas and dots indicate respective sites of pain resulting from distention and contraction of this portion of the duodenum.

For when balloons were lowered into the stomach and duodenum and inflated with air, the patients described the pain as being deep-seated, yet as always lying under the anterior wall between the xiphoid process and the umbilicus. 
In the experiments with the stomach, their results differed from ours chiefly in the fact that the site of distress was not sharply localized, the patient referring to the area by placing his whole hand over the midepigastrium instead of pointing to an area with his finger. Also the sensations were less definable and were related to symptoms arising from overloading the stomach rather than to colic. Presumably the larger area of referred pain was directly related to the fact that the area of stomach wall subjected to pressure by the balloon ( 200 to $500 \mathrm{cc}$. air) was much greater than that subjected to faradic excitation. Distention of the duodenum, on the other hand, resulted in much more definitely localized pain (Figures 10 and 11) than in the experiments with the stomach. We may infer that this was due to the smaller size of the balloon ( 40 to $200 \mathrm{cc}$. air). Even so, the areas pointed to after distention were somewhat larger than those pointed to after faradic stimulation. Yet in neither case did maximum muscle tension cause the pain to be referred to the sides or back of the trunk. The latter phenomenon, when observed clinically, must therefore be due to extension of the lesion into the mesenteries or retroperi. toneal tissues.

The third point, as to why localization of such pain is variable-being felt sometimes at one, sometimes at another portion of the anterior abdominal wall-may be explained, in part, by Sherrington's demonstration of the overlapping of sensory fields in the trunk (see Ranson (9), p. 59).

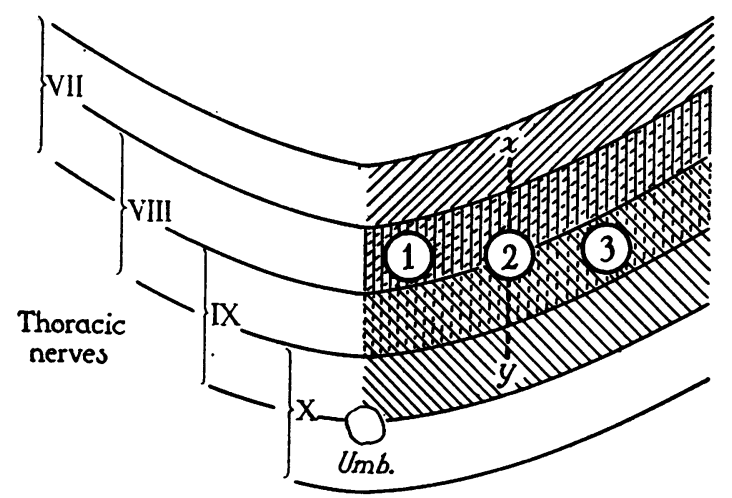

Fig. 12. Diagram (after Ranson, from Sherrington) Illustrating the Overlapping of Sensory Fields of the Abdominal Wall

Vertical lines, zone of thoracic nerve VIII; upper oblique lines, zone of nerve VII; lower oblique lines, zone of nerve IX; Umb., umbilicus. Area 1 supplied by nerves VII and VIII; 2, by VII, VIII, IX; 3, by VIII and IX. (For explanation of $x$ and $y$, see below.)

As shown in Figure 12, the zones of the intercostal nerves dovetail in such a way that Area 1, for instance, is supplied by afferent neurones from both the seventh and eighth, Area 2 from the seventh, eighth and ninth, 
and Area 3 from the eighth and ninth thoracic nerves. As related to faradic stimulation of the stomach, this means that when a subject has localized pain at Area 2 the impulses from the gut may have entered the cord from splanchnic nerves VII, VIII, or IX, or from all three. If the impulse through VII predominated, the pain might have been referred to Area $x$ instead of Area 2 ; or if that through IX predominated it might have been referred to Area $y$ instead of Area 2; but if all three were approximately equal, pain from the stomach might have been localized anywhere from costal margin to umbilicus.

Variability similar to that which we encountered, has also been recorded by Bloomfield and Polland (10), in experimental distention of the duodenum (see Figure 11). Rivers (11), also, seems to have been faced with the same problem, for in certain gastric and duodenal ulcers he found that pain was usually located to the left or right of the umbilicus, whereas in others it shifted to the left or right costal borders, respectively. He would interpret this on the basis of the depth of the lesion, the implication being that different nerve endings of the gut are involved in these two types of cases. Occasionally, also we have noted that increasing the duration of the stimulus (Experiments $4 \mathrm{~b}$ vs. 4c, Figure 4 ) or increasing its strength ( 5 a vs. 5 e, Figure 6 ) has changed the site of pain; but in these cases the presumptively deeper penetration of the current caused the pain to appear at the umbilicus, and the lesser penetration at the costal border-just the reverse of Rivers' findings. Then there is the peculiar situation shown in Figure 7 where the site of pain remained at the umbilicus regardless of the changing nature of the pain, the shifting of the body, or even the shifting of the electrode.

Apparently, therefore, there is some other factor of selection that must be reckoned with. Thus Polland and Bloomfield (12) in their experiments with distention of the esophagus have shown that there are sites of predilection which are not related to the position of the balloon; for out of 191 times in which the tube was inflated just enough to produce a minimal stimulus, pain was localized 87 times at the lower end of the sternum and 48 times just above the suprasternal notch-regardless of the part of the esophagus in which the balloon was situated. Further inflation caused the pain to spread widely or to appear in a new site. Also they found, as we did, that sometimes a constant stimulus in the same individual gave different results.

Similarly, Weiss and Davis (5) have described a typical case in which distention of the lower third of the esophagus caused severe pain between the shoulder blades at the level of the 6th thoracic vertebra. After infiltrating this area of skin with novocain, pain appeared over the 7 th thoracic vertebra; when the latter area was infiltrated, pain appeared over the 4th thoracic vertebra. Finally when this area was injected with novocain (all 
the previous areas being analgesic) slight pain was still felt in the back, but severe pain was felt, anteriorly, over the sternum.

What is the nature of this order of selection? Is it primarily mechanical, depending upon the juxtaposition of nerve endings in the gray matter of the nervous system; or quantitative, depending upon the number of nerve endings from a given nerve at the point of stimulation; or physiological, depending upon such factors as threshold and Bahnung? Or is it a combination of one or more of these factors? The impression that we have gained from these experiments is that it is more than all these and that it involves an integrative process going on in the higher centers.

This brings us to the fourth and fifth points raised by our experiments - namely as to why projection of pain on the abdominal wall tends to follow the course of the gut and yet why, at certain times, it stays in one area regardless of shift of posture or regardless of which segment of the gut is being stimulated.

Unless one accepts the parietocutaneous theory of Morley (vide infra), the interpretation that would seem to fit all the facts most closely is that localization of pain arising from tension of the visceral musculature is the result of integration-a "putting together" by the higher centers of two sources of information, one coming from visceral neurones $a$ (Figure 9) and the other from somatic neurones $c$. This implies a training from birth in the association of impulses carrying true visceral pain with those that are projected from adjacent areas of the skin. Also, if integration be admitted, then we can explain, on grounds of conditioning, such otherwise inexplicable phenomena as have been recorded in Figure 7.

Regarding the latter case it might be said that when localization occurred in the umbilical region, it represented true visceral (protopathic) pain; but when it was localized in the left epigastric region it was due to a spread of the current to the peritoneum of the anterior abdominal wall,from which point it was referred to the overlying skin by a parietocutaneous radiation (see Morley's interpretation of the two kinds of pain in appendicitis).

However, in addition to the reasons already given for believing that the current does not spread from the inside of the gut to the anterior peritoneum (p. 838) there are experimental grounds for questioning Morley's hypothesis. For instance, when he repeated the work of Weiss and Davis he found that while spontaneous pain and hyperalgesia were abolished by intradermal injections of novocain, deep tenderness, pain on coughing and muscle rigidity remained (Morley (13)). Obviously, deep tenderness in these cases could not have been due to a parietocutaneous radiation, otherwise it would have been modified by cutaneous anesthesia. Especially noteworthy was Case 4, of Morley's series, in which the appendix was retrocecal in position and therefore not in contact with the peritoneum of the anterior wall. So also with his ulcer experiments: anatomically, it is 
impossible, by pressing upon the abdominal wall, to bring that portion of it that lies immediately over the pylorus into contact with the duodenal cap. The liver intervenes. Therefore, if the pain of deep tenderness in such cases was not felt directly in parietal nerve endings, it must have arisen in the mesenteries (Sheehan (14)), or in retroperitoneal tissues of embryologically adherent mesenteries, and so have passed into the cord via the splanchnic nerves (e.g., neurone $d$, Figure 9).

Furthermore, it has never been proven experimentally that nerves of the anterior parietal peritoneum do not register pain directly, i.e., without the intervention of Morley's parietocutaneous radiations. On the contrary, Capps and Coleman (4) have reported that when the anterior peritoneum is pricked by wires inserted through trochars embedded in the abdominal wall, the sharp stitch-like pains are localized within half an inch of the end of the wire. (Incidentally, this area of skin should be injected with novocain to ascertain whether the skin is involved at all in this type of pain.) Also there is some evidence that different portions of the peritoneum behave differently. Thus Capps and Coleman have noted that when they touched the periphery of the diaphragm with a wire, the pain was quite different than before; for it became diffuse and was indicated by the patient's placing his hand over the hypochondrium.

The sixth and seventh points raised by our experiments-namely as to why the site of pain migrates from an area of skin that has been anesthetized and simultaneously persists under this area-are best explained by reference to Figure 9 . One must assume that when the cutaneous endings of neurones $c$ are anesthetized, the conductivity or thresholds of these neurones are sufficiently changed (be it ever so slightly) as to eliminate them from competition and to give precedence to adjacent neurones that are simultaneously being bombarded by splanchnic impulses. For example, suppose that Area 2 (Figure 12), which is supplied by peripheral nerves VII, VIII and IX, is anesthetized. Then the pain arising from bombardment of nerves VII, VIII and IX could appear at $x$ or $y$ or any other portion of the skin supplied by the anterior rami of these nerves.

The persistence of pain under the analgesic area may be explained in four ways : 1 -by the bombardment in the ganglia of peripheral neurones coming from deeper layers of the abdominal wall than neurones $c$ (this is not considered probable in view of the experiments of Weiss and Davis); 2-by the continuance of impulses from the cell bodies of neurones $c$, it being assumed that anesthetizing their cutaneous endings may have altered but not abolished the conductivity of these neurones; 3 -by the continuance of impulses from neurones $a$ which had previously conditioned the higher centers to associate the sensation of visceral pain with the area now anesthetized; or 4-by impulses from neurones $a$ which are directly felt in the hollow viscera.

That true visceral pain may persist in the absence of peripheral im- 
pulses is clear from the experiments of Davis, Pollock and Stone (15). These investigators found that after section of all thoracic nerves in cats, the animals still gave evidence of pain when the gallbladder was distended experimentally. It seemed to them, however, that the nature of the pain was somewhat modified. This was not true in our experiments. Therefore one would like to know whether, under such conditions, the localization of pain was modified. Perhaps such needed information may be obtained through the cooperation of patients whose intercostal nerves have been sectioned by thoracoplasty.

Finally, the experiments of Weiss and Davis (5) suggest that pain arising from inflammatory lesions of the gut may have a different mechanism from that arising from excessive muscle tension of the gut. Thus they found that in such inflammatory conditions as acute appendicitis, cholecystitis, etc., spontaneous pain and hyperalgesia were abolished by intradermal injections of novocain. This suggests that what they accomplished by local anesthesia was interference with viscerocutaneous reflexes and not with viscerocutaneous radiations (as in our experiments).

Returning to Figure 9 one may postulate that a visceral afferent impulse arising in one of the tunics of a diseased organ, perhaps in the arteries of that organ (Moore and Singleton (16)), would pass to the lateral column of the cord (neurone $x$ ), then be shunted out to a sympathetic ganglion (neurone $y$ ) and then be relayed to the skin (neurone $z$ ) where it would set up disturbances in the endings of parietal nerves (neurone $c^{\prime}$ ). Thus pain from an inflamed organ might produce superficial tenderness and appear to come from the skin, yet be abolished by anesthetizing the cutaneous endings of postganglionic fibers (neurone $z$ ).

This is the theory of Verger (17), whereby an algogenic stimulus arising in the viscera produces a vasomotor reflex that modifies the "vascular bouquet " of the skin, thereby exciting sensory endings in the skin that are supplied by cerebrospinal nerves. A somewhat comparable theory has been proposed by Sfameni and Lunedei (18). This postulates that algogenic impulses arising in the viscera stimulate efferent neurones in the cord that terminate within sensory corpuscles of the skin,- -the so-called " apparatus of Timofeew" (Maximow (19)), thereby setting up physicochemical changes in the cerebrospinal components of the corpuscle. Such theories as these remove the $a$ priori objections of Morley to viscerocutaneous reflexes and radiations and would seem to open the door to a more neurological approach to the problem of visceral pain.

\section{SUM MARY}

1. An experimental method has been devised whereby the gut may be stimulated, electrically, through the metal end of a stomach tube.

2. As observed under the fluoroscope, excitation with a tetanizing current usually causes ring contraction of the stomach and duodenum. 
3. Such spastic contraction is accompanied by sensations ranging from barely perceptible feelings of pressure to severe colicky pain.

4. Such sensations are definitely localized under the upper quadrants of the abdomen-the subject pointing to the spot with his finger.

5. As the electrode is drawn up through successive portions of the duodenum and stomach, the sites of pain progressively outline the position of these organs; but there is considerable aberrancy (Figures 1 and 2).

6. When the electrode is kept in one segment of the gut, but the body posture is changed, the site of pain usually shifts with it (Figure 3). Sometimes, however, the pain remains localized in one region after both the electrode and the body posture have been changed (Figure 7). This is interpreted as a temporary conditioning of the nervous pathways.

7. When an area of the skin to which the patient has pointed is anesthetized, the pain migrates to a position outside the area, thus revealing that cutaneous nerves are involved in spastic contraction of the gut.

8. These experiments are interpreted to mean that localization of visceral pain arising from spastic contraction of the gut is a viscerocutaneous radiation due to splanchnic bombardment of somatic neurones.

9. At the same time that the pain migrates from an analgesic area it continues to be felt under that area. The similar persistence of visceral pain in animals after section of all thoracic nerves (Davis, Pollock and Stone) suggests that perhaps visceral pain is normally an integration of impulses from both splanchnic and cutaneous sources and explains why such pain tends to follow the course of the gut. Confirmation of this theory awaits experiments with patients in whom the thoracic nerves have been cut.

10. The experiments of Weiss and Davis in abolishing pain from diseased viscera by anesthetizing a localized cutaneous area suggest that the mechanism of pain arising from inflammation may be different from that caused by spastic contraction or distention of the gut. Their work points to an excitation of the skin by reflexes (originating in the viscera) which in turn set up centripetal impulses in the cutaneous endings of peripheral nerves. On the other hand, Morley's inability to abolish deep tenderness by cutaneous anesthesia suggests that such pain is not a parietocutaneous radiation but parietal pain localized in situ or else pain arising in mesenteries or gut which is being transmitted to the cord by splanchnic nerves.

\section{BIBLIOGRAPHY}

1. Boyden, Edward A., and Rigler, Leo G., A cholecystographic and fluoroscopic study of the reaction of the human gall bladder to faradic stimulation of the stomach and duodenum. Anat. Rec., 1934, 59, 427.

2. Moody, R. O., Chamberlain, W. C., and Van Nuys, R. G., Visceral anatomy of healthy adults. Am. J. Anat., 1926, 37, 273.

3. Morley, John, Abdominal pain. E. and S. Livingstone, Edinburgh, 1931. 
4. Capps, Joseph A., and Coleman, George H., An experimental and clinical study of pain in the pleura, pericardium and peritoneum. Macmillan Co., New York, 1932.

5. Weiss, Soma, and Davis, David, The significance of the afferent impulses from the skin in the mechanism of visceral pain. Skin infiltration as a useful therapeutic measure. Am. J. Med. Sc., 1928, 176, 517.

6. Ryle, J. A., An address on visceral pain and referred pain. Lancet, 1926. 1, 895.

7. Lemaire, A., Les douleurs viscérales. Rev. méd. de Louvain, 1926, 81, 129. (Cited by Morley '31.)

8. Dogiel, H. S., Der Bau der Spinal ganglien des Menschen und der Saügetiere. Fischer, Jena, 1908.

9. Ranson, Stephen W., The anatomy of the nervous system. Saunders, Philadelphia, 1928, 3d ed., pp. 59 and 65.

10. Bloomfield, A. L., and Polland, W. S., Experimental referred pain from the gastro-intestinal tract. Part II. Stomach, duodenum and colon. J. Clin. Invest., 1931, 10, 453.

11. Rivers, Andrew B., Pain of peptic ulcer. Northwestern Med., 1934, 33, 6.

12. Polland, W. S., and Bloomfield, A. L., Experimental referred pain from the gastro-intestinal tract. Part I. The esophagus. J. Clin. Invest., 1931, 10, 435.

13. Morley, John, The significance of the afferent impulses from the skin in the mechanism of abdominal pain. Lancet, 1929, 2, 1240.

14. Sheehan, Donal, The afferent nerve supply of the mesentery and its significance in the causation of abdominal pain. J. Anat., 1933, 67, 233.

15. Davis, Loyal, Pollock, Lewis J., and Stone, Theodore T., Visceral pain. Surg., Gynec. and Obst., 1932, 55, 418.

16. Moore, Robert M., and Singleton, A. O., Jr., Studies on the pain sensibility of arteries. II. Peripheral paths of afferent neurones from the arteries of the extremities and of the abdominal viscera. Am. J. Physiol., 1933, 104, 267.

17. Verger, H., Sur une modification due scheme de Lemaire pour la conception physiologique de réflexe viscuso-sensitil de Mackenzie. Gaz. de Sc. méd., 1927, 43, 419.

18. Sfameni, P., and Lunedei, A., Sui riflessi viscero-cutanei e sul meccanismo di produzione del dolore nelle affezioni dei visceri e delle sierose. Riv. di clin. med., 1927, 28, 758.

19. Maximow, Alexander A., and Bloom, William, A textbook of histology. Saunders, Philadelphia, 1930, pp. 283-4. 